\title{
Predictive role of neutrophil to lymphocyte ratio and mean platelet volume in Henoch-Schönlein purpura related gastrointestinal and renal involvement
}

\author{
Halil I. Yakut, Assoc Prof. ${ }^{a}$, Tuba Kurt, MD. ${ }^{b}$, Nermin Uncu, MD. ${ }^{b}$, F. Semsa Cayci, Assoc Prof. ${ }^{c}$ and \\ Banu Celikel Acar, Prof. ${ }^{b}$
}

\begin{abstract}
Background: Henoch-Schönlein purpura (HSP) is a systemic small-vessel vasculitis that occurs mainly in children. The aim was to evaluate the blood neutrophil-to-lymphocyte ratio (NLR) and mean platelet volume (MPV) in patients with HSP and to investigate the relationship with gastrointestinal and renal involvement.

Methods: Children with HSP and healthy individuals as controls were included. Hemoglobin level, white blood cell count, platelet count, NLR, MPV erythrocyte sedimentation rate and C-reactive protein were evaluated.

Results: There were 71 HSP children and 74 controls. NLR was significantly higher in HSP patients with gastrointestinal bleeding than without gastrointestinal bleeding $(p<0,001)$. The optimal cutoff value of NLR for predicting gastrointestinal bleeding was 2.05, with $93 \%$ sensitivity and $62 \%$ specificity. MPV was significantly higher in HSP patients with renal involvement than without renal involvement $(p=0,027)$.

Conclusions: Blood NLR and MPV may be useful markers to identify gastrointestinal and renal involvement in HSP patients. Key words: blood neutrophil-to-lymphocyte ratio, gastrointestinal hemorrhage, Henoch-Schönlein purpura, mean platelet volume, kidney disease.
\end{abstract}

http:/ / dx.doi.org/10.5546/ aap.2020.eng.139

To cite: Yakut HI, Kurt T, Uncu N, Cayci FS, Celikel Acar B. Predictive role of neutrophil to lymphocyte ratio and mean platelet volume in HenochSchönlein purpura related gastrointestinal and renal involvement. Arch Argent Pediatr 2020;118(2):139-142.

a. Department of Pediatrics.

b. Department of Pediatric Rheumatology.

c. Department of Pediatric Nephrology.

University of Health Sciences, Ankara City Hospital,

Ankara, Turkey

E-mail addres:

Banu Celikel Acar: banuacar@gmail.com

Funding: None.

Conflict of interest: None.

Received: 5-29-2019

Accepted: 9-23-2019

\section{INTRODUCTION}

Henoch-Schönlein purpura (HSP) is the most common systemic small-vessel vasculitis in children. Generally, the prognosis of HSP in children is favorable, but there are severe complications such as severe gastrointestinal (GI) involvement in the early phase of disease. ${ }^{1,2}$ The long-term morbidity of HSP is related to the degree of renal involvement. ${ }^{3-5}$ Thus, it is important to assess the risk factors for developing renal involvement in childhood HSP.

Blood neutrophil-to-lymphocyte ratio (NLR) was investigated in many diseases as a marker of inflammation. ${ }^{6-8}$ Few studies on HSP have reported the association of NLR with GI bleeding because of neutrophil dominated inflammation in HSP. In addition, some reports indicate that low mean platelet volume (MPV) is also related to the severity of GI involvement. ${ }^{9}$ NLR and MPV are identified as potentially useful markers of developing GI involvement in early phase of disease.

The aim of our study was to investigate the relationship between NLR and MPV in GI and renal involvement in HSP, which determine the prognosis in long term follow up.

\section{PATIENTS AND METHODS}

All patients diagnosed as HSP between January 2015 and 2018 in our center were reviewed. The study included pediatric patients diagnosed with HSP and healthy children. The European League against Rheumatism/Paediatric Rheumatology European Society (EULAR/ PRES) diagnostic criteria for HSP ${ }^{10}$ was used to define the disease. Control group was selected from age- and sex-matched healthy children who attended the pediatric clinics of our hospital for other reasons.

Exclusion criteria of patients were as follows: [1] with underlying diseases; [2] on medication prior to blood sampling; [3] lacking laboratory data; [4] with risk factors (smoking, obesity, hypertension, diabetes mellitus, etc.) that may affect the outcome of MPV. 
Demographic data, signs and symptoms of disease and laboratory data were noted using the electronic patient database of the time of diagnosis. NLR was calculated as a simple ratio between the absolute neutrophil count and absolute lymphocyte count. Laboratory results of admission (one week time after presentation) were used.

Gastrointestinal bleeding was defined as occult blood in stool, melena, or hematochezia. Renal involvement was defined as the presence of hematuria ( $>5$ red blood cells per microscopic field) and / or proteinuria (urine protein / creatinine ratio $<0.2$ in children $\geq 2$ year of age).

Ethical approval for the study was obtained from the hospital's local ethics committee $(2019 / 20)$, and informed consent was obtained from all patients.

\section{Statistical analysis}

All data were analyzed using Statistical Package for Social Sciences 17.0 (SPSS, Inc. Chicago, Illinois, USA) for windows. Quantitative variables were expressed as mean $\pm S D$ for normally distributed data and as medians (IQR) for non-normally distributed data. Quantitative data for dual groups were compared by using the Student's $t$ test or Mann-Whitney $U$ test when appropriate, whereas $\chi^{2}$ test was performed for qualitative data. Backward stepwise logistic regression analysis was performed to determine the variables associated with GI bleeding and renal involvement in patients with HSP. ROC analysis was used to determine the association of NLR with GI bleeding in HSP; $p$ value $\leq 0.05$ was considered as significant.

\section{RESULTS}

There were 71 participants in the patient group and 74 participants in the control group. There were $41(57.7 \%)$ boys and $30(42.3 \%)$ girls with HSP and $38(51.3 \%)$ boys and $36(48.7 \%)$ girls in the control group. The statistical analyses revealed that the sex distribution between groups was similar $(p=0.506)$. Mean age of the HSP patients was $8.7 \pm 3.6$ years and controls were $9.1 \pm 3.7$ years $(\mathrm{p}=0.472)$.

All the patients had palpable purpura. 58 patients $(81.6 \%)$ had arthritis or arthralgia. $49(69 \%)$ had GI involvement; $16(22.5 \%)$ of these patients had GI bleeding. Two patients presented with intussusception; 22 patients (30.9\%) had renal involvement and of them, 13 patients $(18.3 \%)$ had proteinuria and 9 patients $(12.6 \%)$ had nephrotic-range proteinuria. At first, there were significant differences between HSP patients and healthy controls in terms of white blood cell count (WBC), platelet count (PLT), NLR, MPV, erythrocyte sedimentation rate (ESR), C-reactive protein test (CRP) (Table 1).

We divided the 49 HSP patients with GI involvement into two groups according to presence of GI bleeding. There were 16 patients with GI bleeding and 33 patients without GI bleeding. There were no significant differences regarding WBC, PLT, MPV, ESR and CRP levels between these two groups. However, NLR was significantly higher in HSP patients with GI bleeding than without GI bleeding $(4.7 \pm 3.1$ vs. $2.5 \pm 2.0 ; \mathrm{p}<0.001)$ (Table 2).

Logistic regression analysis of WBC, PLT, MPV, NLR, ESR and CRP were performed to identify the predictive factors for GI bleeding

TABLE 1. Comparison of laboratory parameters between HSP and healthy controls

\begin{tabular}{|c|c|c|c|}
\hline Parameter & $\begin{array}{l}\text { HSP patients } \\
\quad(n=71) \\
\text { mean } \pm S D\end{array}$ & $\begin{array}{l}\text { Healthy controls } \\
\qquad(\mathrm{n}=74) \\
\text { mean } \pm S D\end{array}$ & $p$ value \\
\hline $\mathrm{Hb}(\mathrm{g} / \mathrm{dl})$ & $13.0 \pm 1.0$ & $13.5 \pm 1.2$ & 0.129 \\
\hline WBC (fL) & $12.1 \pm 4.7$ & $8.2 \pm 2.3$ & $<0.001$ \\
\hline PLT (x103/uL) & $393.8 \pm 110.4$ & $319.1 \pm 78.9$ & $<0.001$ \\
\hline NLR & $3.0 \pm 2.4$ & $1.9 \pm 1.8$ & $<0.001$ \\
\hline MPV & $7.7 \pm 0.7$ & $7.9 \pm 0.8$ & $<0.001$ \\
\hline $\mathrm{ESR}(\mathrm{mm} / \mathrm{h})$ & $26.6 \pm 16.0$ & $10.6 \pm 5.7$ & $<0.001$ \\
\hline CRP (mg/dl) & $2.2 \pm 2.7$ & $0.4 \pm 0.5$ & $<0.001$ \\
\hline
\end{tabular}

HSP; Henoch Schonlein purpura, Hb: hemoglobin, WBC: white blood cell, PLT: platelet, NLR: neutrophil-to-lymphocyte ratio, MPV: mean platelet volüme, ESR: erythrocyte sedimentation rate, CRP: C reactive protein. 
in HSP patients. The analysis showed that only NLR was associated with GI bleeding $(\mathrm{p}<0.001)$. ROC analysis was used to define the diagnostic thresholds of NLR for predicting GI bleeding in HSP patients. The area under the curve of NLR was 0.798 (95\% CI 0.66-0.92, $\mathrm{p}<0.001)$ (Figure 1 ). The optimal cutoff value of NLR for predicting GI bleeding was 2.05, with $93 \%$ sensitivity and $62 \%$ specificity, respectively. When the cutoff value of NLR was selected as 2.8 , the sensitivity decreased to $81 \%$ and specificity increased to $77 \%$.

We divided HSP patients into two groups according to the existence of renal involvement. There were 22 patients with renal involvement and 49 patients without renal involvement. There were no significant differences regarding WBC, PLT, NLR, ESR and CRP levels between these two groups. However, MPV was significantly higher in HSP patients with renal involvement than without renal involvement $(8.0 \pm 0.8$ vs. $7.5 \pm 0.7$; $\mathrm{p}=0.027)$ (Table 3).
FIGURE 1. ROC curve of NLR for GI bleeding in HSP

\section{ROC Curve}

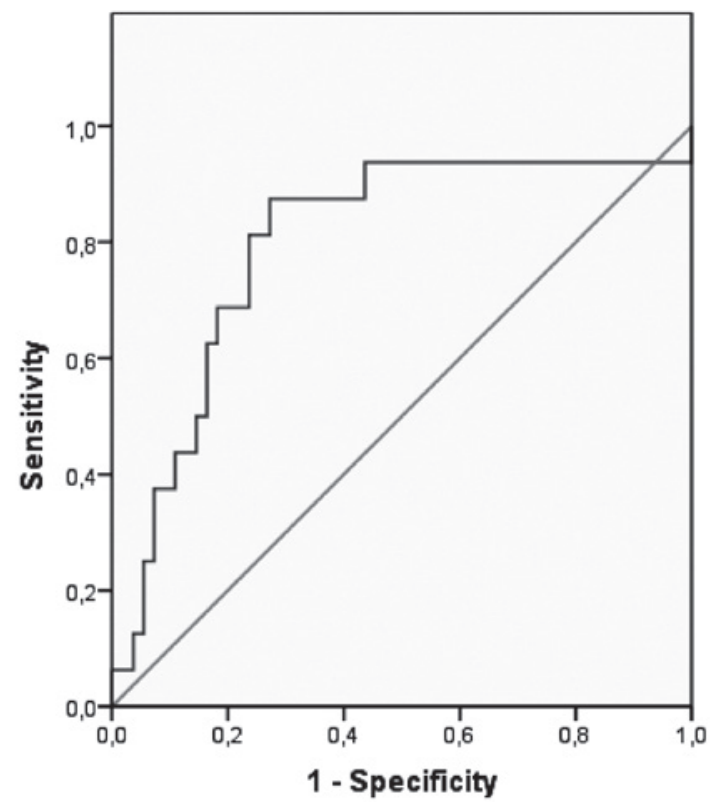

TABLE 2. Comparison of laboratory parameters between patients with and without GI bleeding

\begin{tabular}{lccc}
\hline Parameter & $\begin{array}{c}\text { Patients with GI bleeding } \\
(\mathbf{n}=\mathbf{1 6}) \\
\text { mean } \pm \text { SD }\end{array}$ & $\begin{array}{c}\text { Patients without GI bleeding } \\
(\mathbf{n}=\mathbf{3 3}) \\
\text { mean } \pm \text { SD }\end{array}$ & $p$ value \\
\hline $\mathrm{Hb}(\mathrm{g} / \mathrm{dl})$ & $12.4 \pm 0.9$ & $13.2 \pm 1.0$ & 0.002 \\
$\mathrm{WBC}(\mathrm{fL})$ & $12.7 \pm 4.0$ & $11.9 \pm 4.9$ & 0.198 \\
$\mathrm{PLT}\left(\mathrm{x} 10^{3} / \mathrm{uL}\right)$ & $404.4 \pm 116.4$ & $390.8 \pm 109.5$ & 0.625 \\
$\mathrm{NLR}$ & $4.7 \pm 3.1$ & $2.5 \pm 2.0$ & $<0.001$ \\
$\mathrm{MPV}$ & $7.5 \pm 0.4$ & $7.7 \pm 0.8$ & 0.06 \\
$\mathrm{ESR}(\mathrm{mm} / \mathrm{h})$ & $25.2 \pm 16.6$ & $27.0 \pm 3.2$ & 0.757 \\
$\mathrm{CRP}(\mathrm{mg} / \mathrm{dl})$ & $2.1 \pm 3.2$ & $2.2 \pm 2.6$ & 0.645 \\
\hline
\end{tabular}

GI: gastrointestinal, $\mathrm{Hb}$ : hemoglobin, WBC: white blood cell, PLT: platelet,

NLR: neutrophil-to-lymphocyte ratio, MPV: mean platelet volüme, ESR: erythrocyte sedimentation rate, CRP: C reactive protein.

TABLE 3. Comparison of laboratory parameters between patients with and without renal involvement

\begin{tabular}{lccc}
\hline Parameter & $\begin{array}{c}\text { Patients with renal involvement } \\
(\mathbf{n}=\mathbf{2 2}) \\
\text { mean } \pm \text { SD }\end{array}$ & $\begin{array}{c}\text { Patients without renal involvement } \\
(\mathbf{n}=\mathbf{4 9 )} \\
\text { mean } \pm \text { SD }\end{array}$ & $p$ value \\
\hline $\mathrm{Hb}(\mathrm{g} / \mathrm{dl})$ & $13.4 \pm 0.69$ & $12.9 \pm 1.2$ & 0.041 \\
$\mathrm{WBC}(\mathrm{fL})$ & $11.1 \pm 4.1$ & $12.5 \pm 4.9$ & 0.250 \\
$\mathrm{PLT}\left(\mathrm{x} 10^{3} / \mathrm{uL}\right)$ & $361.8 \pm 82.7$ & $408.2 \pm 118.7$ & 0.129 \\
$\mathrm{NLR}$ & $2.8 \pm 2.2$ & $3.1 \pm 2.5$ & 0.467 \\
$\mathrm{MPV}$ & $8.0 \pm 0.8$ & $7.5 \pm 0.7$ & 0.027 \\
$\mathrm{ESR}(\mathrm{mm} / \mathrm{h})$ & $28.5 \pm 19.7$ & $25.8 \pm 14.2$ & 0.746 \\
$\mathrm{CRP}(\mathrm{mg} / \mathrm{dl})$ & $2.4 \pm 3.0$ & $2.1 \pm 2.7$ & 0.623 \\
\hline
\end{tabular}

Hb: hemoglobin, WBC: whitebloodcell, PLT: platelet, NLR: neutrophil-to-lymphocyte ratio, MPV: mean platelet volüme, ESR: erythrocyte sedimentation rate, CRP: C reactive protein. 


\section{DISCUSSION}

Henoch-Schönlein purpura is a systemic vasculitis characterized by purpuric skin lesions, gastrointestinal manifestations, arthritis and renal involvement. ${ }^{1,2}$ Many studies suggested that thrombocytosis, leukocytosis, and high ESR, CRP levels were associated with GI bleeding in HSP .,11

In the past few years, it has been revealed that MPV plays an actual role in inflammatory disorders. Shi et al. demonstrated that lower MPV values were observed in children with HSP than healthy individuals. ${ }^{12}$ Makay et al., revealed that low MPV might be related to GI bleeding in HSP. ${ }^{9}$ Some studies suggest that several inflammatory cytokines have been found to be increased in HSP patients and, these inflammatory cytokines are related to platelet activation. Inflammatory cytokines activate megakaryocytes to release more small sized platelets, so increased inflammatory cytokines may lead to a decrease in MPV. Further investigation suggests that large platelets in severe inflammatory diseases are consumed at a greater rate of inflammation sites than small platelets. ${ }^{13,14}$ As a result, it is inevitable that MPV is low to reflect the severity of inflammation. In our study, although we could not determine a cutoff MPV value, MPV was found to be lower in patients with GI bleeding than in non-bleeding patients.

The neutrophil to lymphocyte ratio has been widely used to define the severity of inflammation. ${ }^{6-8}$ Neutrophilia or lymphopenia results in high NLR while lymphocytosis or neutropenia results in low NLR. One of the characteristic histopathological findings in HSP is neutrophilic cell infiltration. The results of our study demonstrated that HSP patients with GI bleeding had significantly higher NLR than those without GI bleeding. In this study, the optimal cutoff NLR for predicting GI bleeding was 2.05 with $93 \%$ sensitivity and $62 \%$ specificity. We suggest that this cutoff NLR may be used to choose HSP patients who will suffer from GI bleeding.

Renal involvement develops within 4-6 weeks of the onset of the rash, but end-stage disease may not be obvious for a number of years. Age at onset of more than 7 years old, severe abdominal symptoms, persistent purpuric lesions, and decreased factor XIII activity are associated with an increased risk of nephritis. ${ }^{15}$ In addition to these, low MPV was found to be a risk factor of renal involvement in our study. But, it should not be forgotten that MPV itself alone without other variables may not provide exact predictive information about GI bleeding in children with HSP.
In our study, however, several limitations exist. Firstly, it should be noted that this study used a relatively small sample size, especially of renal involvement in HSP patients. Secondly, MPV levels may change during in the course of HSP. Finally, this study was retrospective.

In conclusion, NLR was found as significantly increased in GI bleeding. Moreover, lower MPV levels were associated with renal involvement in patients with HSP. Therefore, NLR and MPV may be a useful marker to GI bleeding and renal involvement in HSP patients.

\section{REFERENCES}

1. McCarthy HJ, Tizard EJ. Clinical practice: Diagnosis and management of Henoch-Schönlein purpura. Eur J Pediatr. 2010; 169(6):643-50.

2. Davin JC, Coppo R. Henoch-Schönlein purpura nephritis in children. Nat Rev Nephrol. 2014; 10(10):563-73.

3. Ronkainen J, Nuutinen M, Koskimies O. The adult kidney 24 years after childhood Henoch-Schönlein purpura: a retrospective cohort study. Lancet. 2002; 360(9334):666-70.

4. Stewart M, Savage JM, Bell B, McCord B. Long term renal prognosis of Henoch-Schönlein purpura in an unselected childhood population. Eur J Pediatr. 1988; 147(2):113-5.

5. Lin SJ, Huang JL, Hsieh KH. Clinical and laboratory correlation of acute Henoch-Schönlein purpura in children. Zhonghua Min Guo Xiao Er Ke Yi Xue Hui Za Zhi. 1998; 39(2):94-8.

6. Uslu AU, DeveciK, KorkmazS, Aydin B, etal. Is neutrophil/ lymphocyte ratio associated with subclinical inflammation and amyloidosis in patients with familial Mediterranean fever? Biomed Res Int. 2013; 2013:185317.

7. Kim JY, Park T, Jeong SH, Jeong CY, et al. Prognostic importance of baseline neutrophil to lymphocyte ratio in patients with advanced papillary thyroid carcinomas. Endocrine. 2013; 46(3):526-31.

8. O'Brien CE, Price ET. The blood neutrophil to lymphocyte ratio correlates with clinical status in children with cystic fibrosis: a retrospective study. PLoS One. 2013;8(10):e77420.

9. Makay B, Türkyilmaz Z, Duman M, Unsal E. Mean platelet volume in Henoch-Schönlein purpura: relationship to gastrointestinalbleeding. Clin Rheumatol.2009;28(10):1225-8.

10. Ozen S, Ruperto N, Dillon MJ, Bagga A, et al. EULAR/ PReS endorsed consensus criteria for the classification of childhood vasculitides. Ann Rheum Dis. 2006; 65(7):936-41.

11. Chan H, Tang Y, Lv X, Zhang G, et al. Risk factors associated with renal involvement in childhood Henoch-Schönlein purpura: a meta-analysis. PLoS One. 2016; 11(11):e0167346.

12. Shi X, Li CW, Mo LJ, Li XH, et al. Altered mean platelet volume in children with Henoch-Schönlein purpura and its association with disease activity. Ann Clin Biochem. 2018; 55(3):368-72.

13. Ha TS. The role of tumor necrosis factor-alpha in HenochSchönlein purpura. Pediatr Nephrol. 2005; 20(2):149-53.

14. Kimura S, Takeuchi S, Soma Y, Kawakami T. Raised serum levels of interleukins 6 and 8 and antiphospholipid antibodies in an adult patient with Henoch-Schönlein purpura. Clin Exp Dermatol. 2013; 38(7):730-6.

15. de Almeida JL, Campos LM, Paim LB, Leone C, et al. Renal involvement in Henoch-Schönlein purpura: a multivariate analysis of initial prognostic factors. J Pediatr (Rio J). 2007; 83(3):259-66. 